\title{
Towards an analytical model for characterising behaviour of high-speed VVoIP applications
}

\author{
Andrei Sukhov ${ }^{1}$, Prasad Calyam ${ }^{2}$, Warren Daly ${ }^{3}$, Alexander Ilin ${ }^{4}$ \\ ${ }^{1}$ Laboratory of Network Technologies, Samara Academy of Transport Engineering, \\ 1st Bezymyannyi per., 18, Samara, 443066, Russia \\ e-mail:sukhov@ssau.ru \\ ${ }^{2}$ OARnet, The Ohio State University, 1224 Kinnear Road, Columbus, Ohio 43212, USA \\ e-mail:pcalyam@oar.net \\ ${ }^{3}$ HeaNet Ltd, Crampton Ave, Shelbourne Rd, Ballsbridge, Dublin 4, Ireland \\ e-mail: warren.daly@heanet.ie \\ ${ }^{4}$ Russian Institute for Public Network, Kurchatov sq., 1, Moscow, 123182, Russia \\ e-mail:kalend@ripn.net
}

\begin{abstract}
The use of high-speed multimedia technologies such as MPEG-based interactive Videoconferencing and IPTV for delivering Television content over the Internet is becoming increasingly common. With commercial vendors and content providers envisioning products and services for delivering audiovisual content equipped with greater user-control and higher resolutions, there has arisen a significant demand to identify and quantify various bottlenecks in the Internet to better support such ventures. In this paper, we attempt to develop an analytical model that reflects the effects of various device factors and network factors that affect the performance of highspeed interactive audiovisual streams, i.e. the ultimate end-user perception of audiovisual quality. To develop our model, we use a set of experimental results obtained in both a LAN as well as on an Internet testbed.
\end{abstract}

Key words: videoconferencing, IPTV, network impairments, human factors

\section{INTRODUCTION}

The impetuous growth of traffic volumes of interactive audiovisual applications on the Internet can be attributed to the rapid advances in numerous technologies associated with both network infrastructure and Voice and Video over IP (VVoIP) applications such as Videoconferencing, Video on Demand and IPTV. With end-users demanding superior interactive voice and video communications over the Internet that offers only "best effort service", many studies have been conducted $[4,5,16,18]$ for understanding behaviour and bottlenecks relating to such voice and video traffic to find ways to improve the Internet infrastructure, i.e., the VVoIP system.

These earlier works focused on many aspects of network performance bottleneck issues such as frame rate, packet delay, jitter, loss and reordering mainly in the pur- view of voice traffic and in some cases in the purview of video traffic. While addressing video traffic behaviour, these studies have limited their scope to low-speed interactive audiovisual streams $(<384 \mathrm{Kbps})$ and have failed to verify these findings to be applicable to highspeed interactive multimedia data streams shown in Table 1. Since high-speed VVoIP applications using technologies in Table 1 are beginning to become widespread in use [22], it has become necessary to study performance bottlenecks relating to these applications by extending earlier principles and methodologies to better understand high-speed VVoIP system bottlenecks.

The primary challenges that distinguish the study of high-speed interactive audiovisual streams compared to the study of low-speed interactive audiovisual streams include:

- Understanding the effects of device factors (e.g., compression schemes, jitter buffering schemes, packet loss con-

Table 1. High-speed interactive multimedia data stream technologies

\begin{tabular}{lcc}
\hline Application Video Quality & Technology & Bitrate (Kbps) \\
\hline HDTV, $R \approx 1920 \times 1080, F_{r}=60 \mathrm{fps}, q=32$ & MPEG-2 & 2048 \\
VCR-quality television, $R \approx 400 \times 300, F_{r}=30 \mathrm{fps}$ & MPEG-1 & 1200 \\
Videoconferencing, $R \approx \mathrm{CIF}(352 \times 288), F_{r}=(15-30) \mathrm{fps}, q=16$ & H.261, H.263 & $384-1920$ \\
\hline
\end{tabular}


cealment schemes) to maintain consistent high frame rates on the receiver side in spite of the degradation of stream quality due to network factors (e.g. queuing due to congestion, packet delay, loss and reordering);

- Quantifying end-user perceived experience when there are high expectations of audiovisual quality in correspondence to the effects caused by device and network factors.

In this paper, we address our above challenges by attempting to derive an analytical model that suggests the effects of the network and device factors on the resulting end-user perception (i.e., Mean Opinion Scores) in any given high-speed VVoIP system. We use experimental results from a LAN and an Internet testbed to support our derived model conclusions.

The remainder of this paper is organized as follows: Section 2 describes various terminologies used in our model. Section 3 describes our experimental setup used for data collection. Section 4 details our analytical model and salient observations. Section 5 concludes the paper.

\section{TERMINOLOGY}

In this section, we define various terms used in our analytical model. The significance of a few of the terms

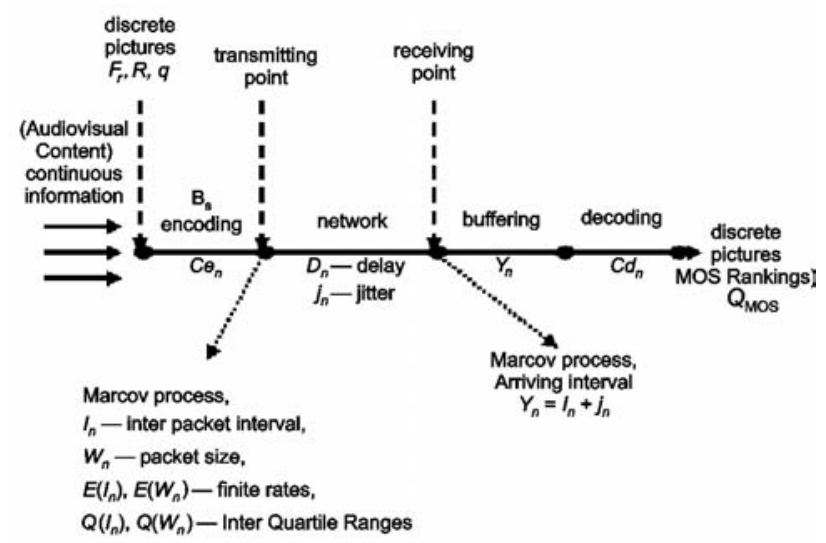

Fig. 1. End-to-end parameters for analytical model

defined in this section are shown as relevant to an actual VVoIP system in Fig. 1.

\subsection{Encoding rate $\left(B_{s}\right)$}

The encoding rate of the video streams is determined by a video codec with Compression factor Cen and Decompression factor $\mathrm{Cdn}$ together with a co-efficient of compression $\tau$. $\mathrm{B}_{\mathrm{s}}$ value also depends on the capabilities of the source (CPU, clock rate, etc.), video content being encoded $\left(A_{1}\right)$ and the access bandwidth to the Internet $K . B_{s}$ can be written as follows

$$
B_{s}=\tau(K) A_{l}\left(F_{r}, R, q\right)
$$

where Frame Rate $\left(F_{r}\right)$ is the number of frames per second during an audiovisual conference connection. Video Resolution $(R)$ is the ability to discriminate closely spaced lines on the receiving screen and is measured in pixels. For example, versions of H.263 support five standardized picture formats: SQCIF $(128 \times 96)$, QCIF $(176 \times 144)$, CIF $(352 \times 288)$, 4 CIF $(704 \times 576)$, and $16 \mathrm{CIF}(1408 \times 1152)$. Color depth $(q)$ is the number of bits used in a frame of video sequence.

\subsection{Inter-packet times $\left(I_{n}\right)$}

It can be defined as the times between successive packets at both the sender and receiver ends. Various parameters associated with inter-packet times are shown in Fig. 1. $T_{n}$ depicts the transmission time at the sender $\left(T_{n}^{s}\right)$ or arrival time at the receiver $\left(T_{n}^{r}\right)$. In depicts the variation between the successive packet transmissions or variations in the received packet arrival times $\left(Y_{n}\right) . W_{n}$ depicts the packet size distribution in the packet sequences. We can also consider $Z_{n}$, which depicts the smoothened inter-packet sequence by the dejitter buffer at the receiver end-point.

\subsection{Network health $\left(N_{h}\right)$}

Network health [11] can be characterised as a function of Delay $\left(D_{n}\right)$, Jitter $\left(j_{n}\right)$ and Loss $(p)$ as given by

$$
N_{h}=f\left(D_{n}, f_{n}, p\right)
$$

where Delay is the amount of time a packet takes to travel from the sender's application to reach the receiver's destination application. Jitter is defined as the variation in the delay of the packets arriving at the receiving end. Loss is defined as the percentage of transmitted packets that never reach the intended destination due to packets discarded deliberately $(\mathrm{RED}, \mathrm{TTL}=0)$ or non-deliberately by intermediate links (layer-1), nodes (layer-3) and endsystems (discard due to late arrivals at the application) along a given transmission path.

\subsection{Audiovisual content $\left(A_{l}\right)$}

$\mathrm{Al}$ corresponds to the various low temporal (e.g., casual videoconference, business presentation) or high temporal (e.g., musical concert or sports broadcast) media streams generated using high-speed VVoIP applications, which need to be encoded/decoded, and transmitted through the network. The perception of the audiovisual content is quantified using the Mean Opinion Score $\left(Q_{\mathrm{MOS}}\right)$ metric which is used to obtain perceptual quality on a scale of 1 to 5 [15], of end-users based on the interaction response times in the system and based on the impairments observed in video streams (e.g., frame freezing, tiling) and audio streams (e.g. echoes, dropouts) [10, 19]. 


\section{TESTBED SETUP}

Figures 2 and 3 show the setup for the experiments in the LAN and Internet environments, respectively. Both testbeds involved obtaining Ethereal traffic traces for low, medium and high temporal audiovisual content at encoding bitrates: 128, 384, 512, 768, 1024, 1472, 1920 Kbps. To extract the necessary data for determining the data distribution for inter-packet intervals $I_{n}$ and packet sizes $W_{n}$ from the traces, we used the RTP analysis module of the Ethereal network protocol analyser. We filtered-out the audio and video streams from the LAN traffic and decoded the audio and video packets information.
The data from the Internet experiments involved testing between RIPN, HEAnet and OARnet. The network paths between the test sites were comprehensively measured using various tools [17] such as $I_{\text {perf }}, H .323$ Beacon [4] and Pathping. All the tools reported excellent network path between the HEAnet and OARnet sites. For example, the H.323 Beacon Server-to-Server test indicated a MOS score of 4.33 with end-to-end jitter less than $j<4 \mathrm{~ms}$ and almost negligible loss $(p<0.12 \%)$. The connection between HEAnet and the Russian site formed the best comparative site for our results, due to its poor network path characteristics. The H.323 Beacon results between the sites indicated $<4.0$ MOS.
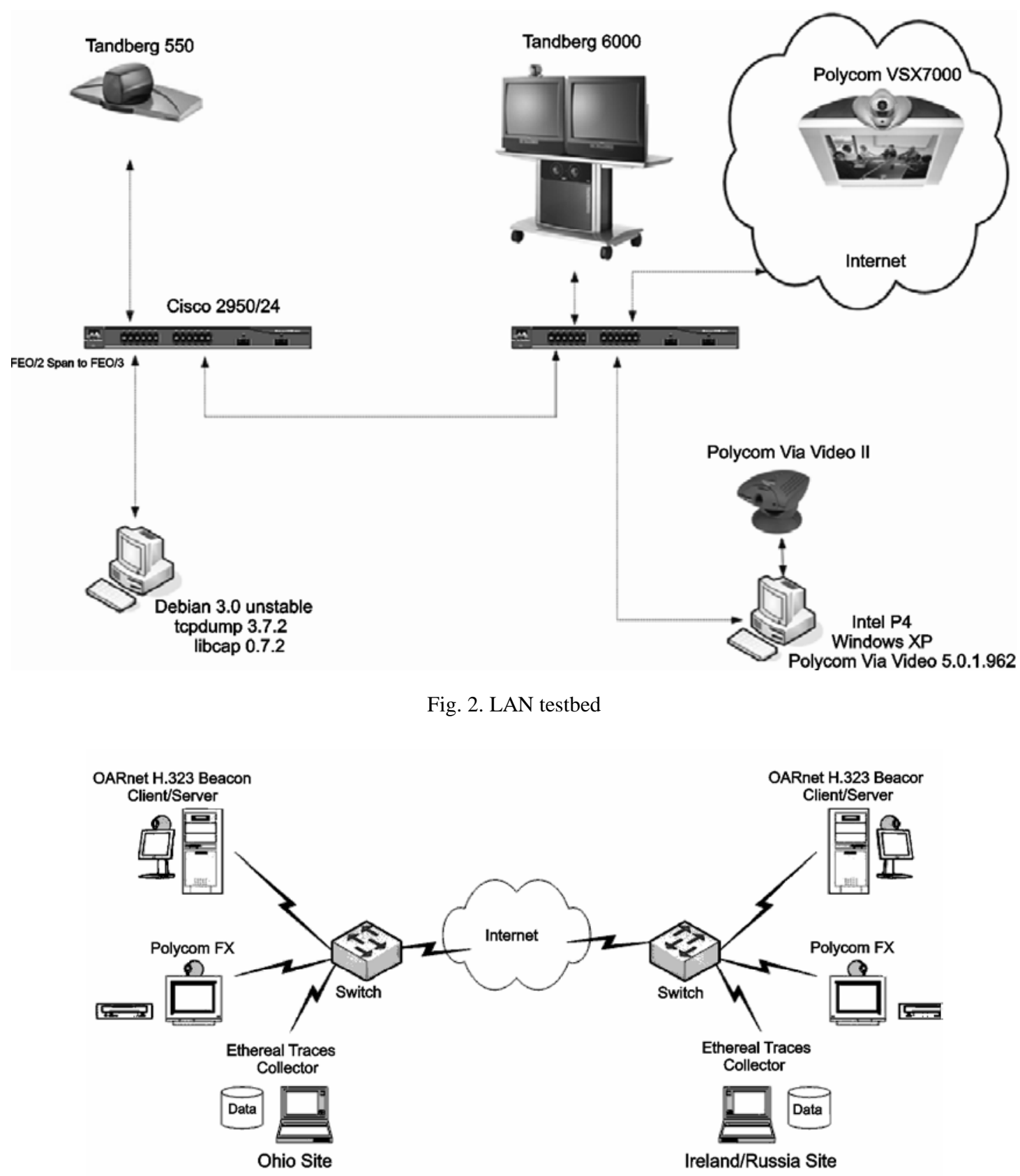

Polycom Via Video 5.0.1.962

Fig. 2. LAN testbed

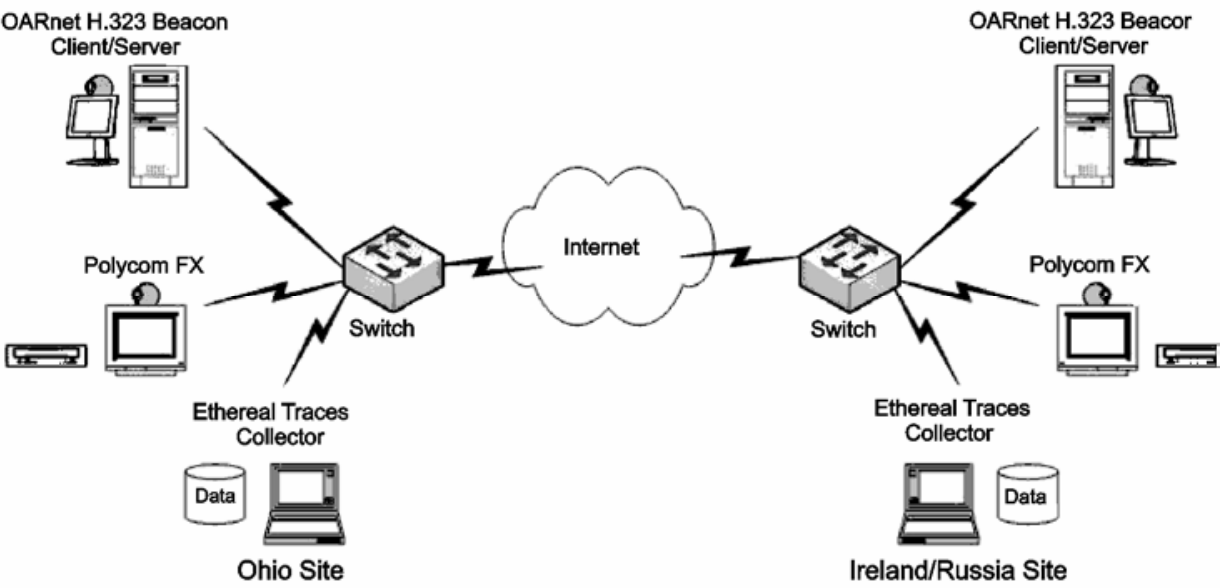

Fig. 3. Internet testbed 


\section{HIGH SPEED VVOIP ANALYTICAL MODEL}

In this section, we describe our analytical model for a high-speed VVoIP system. We use the following assumptions in describing our analytical model $[1,2,20]$ :

- A Markov process [9, 13] can be used to describe the arrival of the Audio and Video packets (RTP packets);

- The distribution of packet size $W_{n}$ and sender side interpacket intervals $I_{n}$ can be characterised by using average values $E\left[I_{n}\right]$ and $E\left[W_{n}\right]$ respectively;

- A function $\varphi$ from Eqn. (3)

$$
\varphi\left(\Delta Q_{\mathrm{MOS}}\right)=E\left[I_{n}\right] / j
$$

describes the relation of network jitter $j$ and sender-side inter-packet time $E\left[I_{n}\right]$ on the end-user perceived audiovisual quality QMOS.

Here $\Delta Q_{\mathrm{MOS}}=Q_{\mathrm{MOS}}^{t}-Q_{\mathrm{MOS}}^{r}$ is the degradation of video quality after transmitting the data over IP network, $Q_{\mathrm{MOS}}^{t}$ and $Q_{\mathrm{MOS}}^{r}$ is the video quality at transmitting and received points correspondingly. The average value of $E\left[I_{n}\right]$ denotes the initial inter-packet jitter introduced by
Using $I_{n}$ and $W_{n}$ data, we can determine the average values: $E\left[I_{n}\right]$ and $E\left[W_{n}\right]$, as well as their Inter Quartile Range (IQR) values: $\Theta\left[I_{n}\right], \Theta\left[W_{n}\right]$. The values observed in our experiments to study the characteristics of $W_{n}$ and $I_{n}$ are shown in Table 2 and in Figs. 4-6.

Figure 4 shows the cumulative number $N$ of RTP (H.261) packets that arrive at the receiver end-point during a 60 seconds interval. The arrival rate $\lambda=1 / E\left[I_{n}\right]$ remains mostly constant throughout the 60 seconds interval. Figure 5 shows typical example of a distribution density of inter-packet intervals In for a video sequence. The local peaks on Fig. 5 corresponds approximately to $0.5,10,20$, 30, $40 \mathrm{~ms}$, etc at connection speeds of $512 \mathrm{Kbps}$ and CIF resolution. The local peaks compare to the Gaussian curves, therefore the distribution density $p^{I}(x)$ can be written as described in [12]

$$
p^{I}(x)=k \sum_{i} A_{i} \exp \left(-\frac{\left(x-I_{i}\right)^{2}}{2 \sigma_{i}^{2}}\right)
$$

Table 2. Values of $\left[W_{n}\right]$ and $\left[I_{n}\right]$ at different video resolutions

\begin{tabular}{lrcccc}
\hline Resolution & $B_{s}, \mathrm{Kbps}$ & $E\left[W_{n}\right]$, bytes & $\Theta\left[W_{n}\right]$, bytes & $E\left[I_{n}\right], \mathrm{ms}$ & $\Theta\left[I_{n}\right], \mathrm{ms}$ \\
\hline QCIF, CIF & 128 & $600-800$ & $600-800$ & $70-100$ & $50-80$ \\
CIF, 4CIF & 384 & $900-1100$ & $500-700$ & $20-30$ & $10-20$ \\
CIF, 4CIF & 512 & $1000-1200$ & $400-600$ & $17-25$ & $10-18$ \\
CIF, 4CIF & 768 & $1000-1200$ & $300-600$ & $12-20$ & $10-15$ \\
CIF, 4CIF & 1024 & $1000-1300$ & $300-600$ & $10-18$ & $8-14$ \\
4 CIF & 1472 & $1000-1300$ & $300-600$ & $8-15$ & $7-14$ \\
4 CIF, 16CIF & 1920 & $1000-1300$ & $300-600$ & $5-10$ & $7-12$ \\
\hline
\end{tabular}

the sender equipment before the audiovisual streams enter the network. Small values of $E\left[I_{n}\right]$ correspond to low jitter and thus a better quality of initial audiovisual streams $\left(Q_{\mathrm{MOS}}^{t}\right)$.

Smaller values of network jitter $j$ correspond to higher values of user-perceived quality rankings $Q_{\text {MOs. Thus the }}$ upper limit of $Q_{\text {MOS }}[6,7]$ for a given experiment with a given audiovisual content and end-to-end network topology determines the resultant $I_{n}$ characteristics and consequently the inter-packet jitter distributions at the receiver end.

In order to ameliorate the distortion of initial sequence, dejitter buffering schemes are applied before the decoding process at the receiver-end. The bound of amelioration is influenced by the value $Y_{\max }$. If the arriving interval $Y_{n}=I_{n}+j_{n}$ (see Fig. 1) or inter-packet Jitter after network distortion exceeds this period $Y_{\max }$ [21] then we experience a packet discard that affects $Q_{\mathrm{MOS}}$ :

$$
Y_{\max }=-\ln P\left(Y_{n} \geq Y_{\max }\right) E\left[I_{n}\right],
$$

where $P\left(Y_{n} \geq Y_{\max }\right)$ is the probability of event when arriving interval exceeds the buffering time equals.

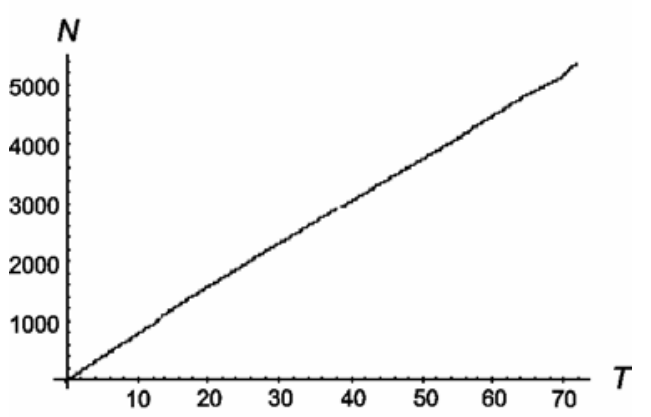

Fig. 4. RTP (H.261) packet arrivals during 60 seconds interval at $768 \mathrm{Kbps}$

Here

- $\quad k$ is the normalizing coefficient, so that $\int_{1}^{\infty} p^{I}(x) d x=1$.

- Values $I_{i}$ describe the local peaks of ${ }^{1} I(x) ; A_{i}$ and $\sigma_{i}$ characterises their respective amplitudes and dispersions.

It should be noted that a voice sequence (Ex., ITU-T G.722) consists of packets of constant packet sizes of 

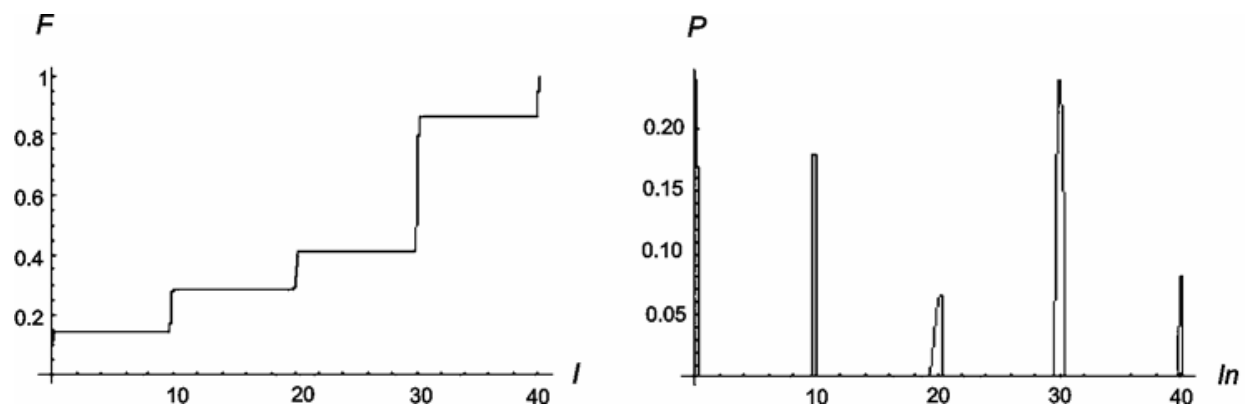

Fig. 5. Typical distribution $F$ and distribution density $P$ of inter-packet intervals $I_{n}$ at $512 \mathrm{Kbps}$

NOTE: For data shown in Fig. 5, the average size of inter-packet interval $E^{512}\left[I_{n}\right]=23.0 \mathrm{~ms}$, IQR value $\Theta^{512}\left[I_{n}\right]=29.7 \mathrm{~ms}$ and quartiles are $\Theta_{1 / 4}^{512}\left[I_{n}\right]=10.4 \mathrm{~ms}, \Theta_{3 / 4}^{512}\left[I_{n}\right]=30.1 \mathrm{~ms}$
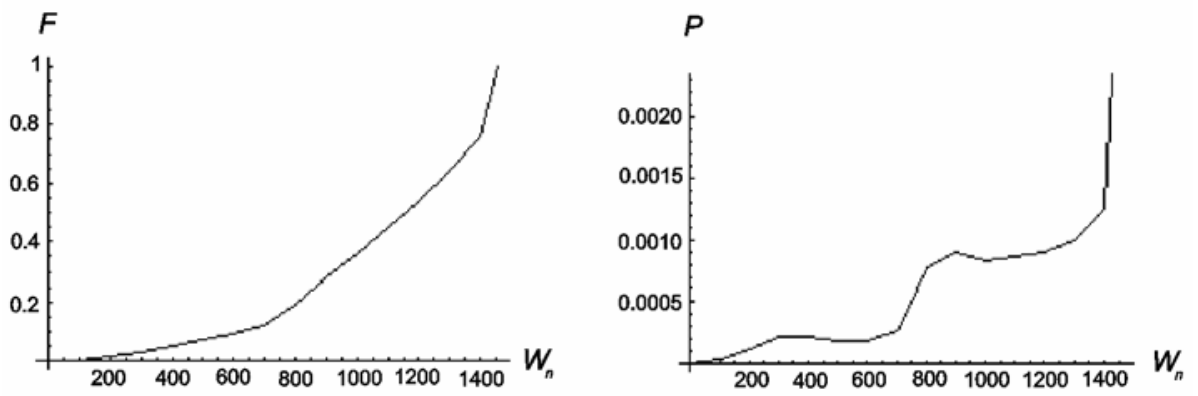

Fig. 6. Typical distribution $F$ and distribution density $p$ of inter-packet intervals $W_{n}$ at $512 \mathrm{Kbps}$ NOTE: For data shown in Fig. 6, the average size of inter-packet interval $\mathrm{E}^{1920}\left[W_{n}\right]=1086$ bytes, IQR value $\Theta^{1920}\left[W_{n}\right]=533$ bytes and quartiles are $\Theta_{1 / 4}^{1920}\left[W_{n}\right]=860$ bytes, $\Theta_{3 / 4}^{1920}\left[W_{n}\right]=1393$ bytes

374 bytes whereas video sequence (Ex., ITU-T H.261) consists of packet sizes varying between 65 bytes to 1518 bytes.

A typical packet size density distribution for H.261 traffic can be studied using Fig. 6. The $x$ axis corresponds to the size of $W_{n}$ in bytes, $y$ axis reflects the relative frequency of such packets in our data. It can be seen that about $25 \%$ of the small packet sizes do not exceed 860 bytes. Thus the video sequence is quite different from a voice sequence in terms of packet sizes and inter-arrival jitter values.

We can express the objective MOS ranking for voice as follows:

$$
\Delta Q_{\mathrm{MOS}}^{\mathrm{voice}}=0.012 \sigma\left(D_{n+1}-D_{n}\right)+0.24 p
$$

where $\sigma\left(D_{n+1}-D_{n}\right)$ is the quadratic mean and $p$ is packet loss value measured in percentage of packets received as result of a ping sequence [8] of more than 100 packets:

$$
\text { ping -s [value }>100 \text { ] [IP address of another end point] }
$$

Since there is no conventional test method for determining objective MOS ranking for video connections, our coefficients in formula for video are purely subjective [3]. Thus the function $\varphi\left(\Delta Q_{\mathrm{MOS}}\right)$ is given by the equation:

$$
\Delta Q_{\mathrm{MOS}}^{\mathrm{video}}=0.015 J+0.008 J-0.0006 J I
$$

where the $J=\sigma\left(D_{n+1}-D_{n}\right)+20 p$ is the cumulative variable described Network Factor and $\sigma, p$ are the results of a ping sequence of more than 100 packets:

$$
\text { ping -s [value }>100 \text { ] [IP address of another end point] }
$$

The value of $I$ is given from the Table 2 .

During signal processing of the audiovisual streams, the transformation of continuous picture in discrete sequence causes an unavoidable deterioration of quality of technical picture in comparison to the original generated streams. Therefore the maximal value $Q_{\mathrm{MOS}}$ reaches upto only 4.41 even in the most ideal conditions. When the connection bitrate becomes smaller than the quantity required for encoding or decoding of the picture, a frame drop is noticed. This fact explains the term $\beta I$ in Eq. (8) when $J=0$. The network induced degradation described by variable $J$ causes drop in the connection video quality more intensely at high bit rates. In other words the variable

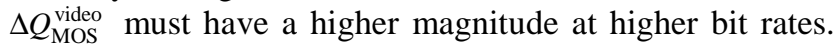
Also, since $J$ and $I$ can be ameliorated at receiver endpoints using packet loss concealment schemes and dejitter buffering schemes, we can express the overall VVoIP model in general as follows: 
Hence, we have

$$
Q_{\mathrm{MOS}}^{\mathrm{video}}=Q_{\text {ideal }}-\alpha J-\beta I+\gamma J I
$$

where:

- $Q_{\text {ideal }}$ is maximum value of multimedia quality obtainable at the sender end-point before ingress into the network. Its value depends on the video and audio codecs used and the audiovisual content generated at the end-point side; - $J=I P D V+\zeta p$ is variable that quantifies Network induced degradation. Here, IPDV is the inter-packet delay variance and $p$ is percent of packet loss in the audiovisual stream;

- I is Inter-packet jiter before network distortion caused by the initial encoding device at the sender side;

- $\alpha, \beta, \gamma, \zeta$ are the numerical coefficients that must be found experimentally for any given path.

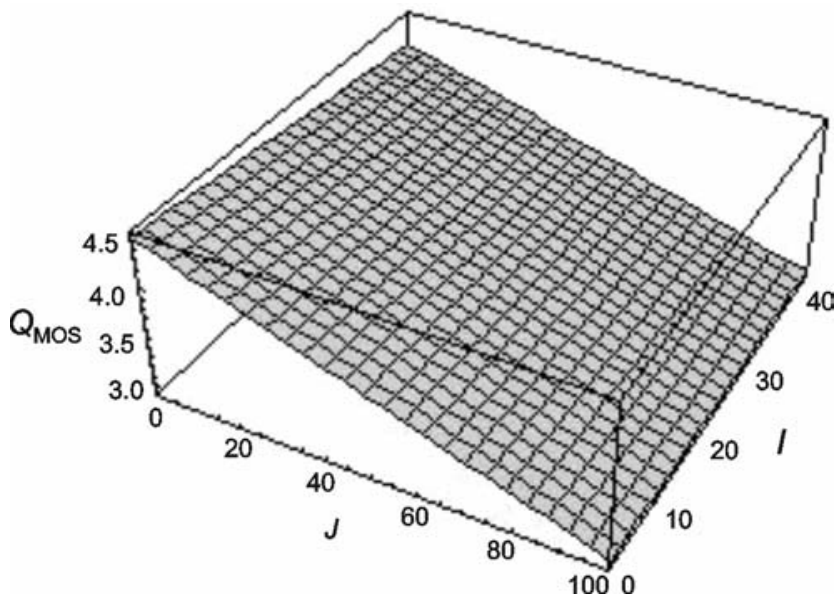

Fig. 7. $3 D$ representation of VVoIP model surface

Our VVoIP model ultimately can be visualized as a $3 D$ surface whose surface characteristics are determined by the values of the numerical coefficients $\alpha, \beta$ and $\gamma$ explained above. Figure 7 shows a typical $3 D$ representation of our VVoIP model with axes -inter-packet jitter before network distortion $(I)$, network induced degradation $(J)$ and the final perceived $Q_{\text {Mos. }}$. Thus, we can imagine a unique surface that overall characterises the human perceived audiovisual quality by taking into account the degradation of the audiovisual streams due to both intermediate encoding/decoding device and network factors and any amelioration that the audiovisual streams can be subjected to, in a given VVoIP system.

\section{CONCLUSION}

In this paper, we presented our measurement methodology to develop a basic analytical model that features the impact and inter-dependencies of various network and device features on the end-user perception of audiovisual quality when using interactive high-speed multimedia applications. We are planning to conduct further tests with more extensive experiments whose results could further strengthen the validity of our analytical model and also could lead us in the direction of extending our proposed analytical model to be more comprehensive in nature.

\section{References}

[1] Ch. Barakat, P. Thiran, G. Iannaccone, Ch. Diot, Ph. Owezarski, A flow-based model for Internet backbone traffic, IEEE Transactions on Signal Processing - Special Issue on Signal Processing in Networking, 51, 8, 2111-2124, August 2003.

[2] S. Ben Fredj, T. Bonald, A. Proutiere, G. Regnie, J. Roberts, Statistical Bandwidth Sharing: A Study of Congestion at Flow Level, ACM SIGCOMM, August 2001.

[3] S. Bonaventura, F. Baldi, M. Alfano, M. Mirabelli, J. Antkowiak, A. Dehnel, Testing user perceived multimedia communication quality using realistic tasks, EURESCOM, Project P807: JUPITER2 - Joint Usability, Performability and Interoperability Trials in Europe, 1999.

[4] P. Calyam, W. Mandrawa, M. Sridharan, A. Khan, P. Schopis, H.323 Beacon: An H.323 application related end-toend performance troubleshooting tool, Proceedings of ACM SIGCOMM Network Troubleshooting Workshop (NetTs' 04), Portland, October 2004.

[5] P. Calyam, M. Sridharan, W. Mandrawa, P. Schopis, Performance Measurement and Analysis of H.323 Traffic, Passive and Active Measurement Workshop (PAM), 2004.

[6] A. Clark, Modelling the effects of burst packet loss and recency on subjective voice quality, 2001.

[7] M. Claypool, J. Tanner, The Effects of Jitter on the Perceptual Quality of Video, ACM Multimedia", 1999

[8] L. Cottrell, W. Matthews, C. Logg, Tutorial on Internet Monitoring $\backslash \&$ PingER at SLAC, 2001, http: //www.slac.stanford.edu/comp/net/wan-mon/tutorial.html

[9] Chen-Nee Chuah and Randy H. Katz, Characterizing Packet Audio Streams from Internet Multimedia Applications, ICC 2002.

[10] R. Finger, A. W. Davis, Measuring Video Quality in Videoconferencing Systems.

[11] F. Fluckiger, Understanding Networked Multimedia, Prentice Hall, Inc. 1995.

[12] B. V. Gnedenko, Theory of Proability, NY, Gordon and Breach Science Publishers, 1999.

[13] W. Jiang and H. Schulzrinne, Modeling of packet loss and delay and their effect on real-time multimedia service quality, Proc. of NOSSDAV, 2000.

[14] ITU-T Recommendation G.107, The Emodel, a computational model for use in transmission planning, 1998.

[15] ITU-T Recommendation P.911, Subjective audiovisual quality assessment methods for multimedia applications, 1998

[16] M. Karam, F. Tobagi, Analysis of the Delay and Jitter of Voice Traffic over the Internet, IEEE INFOCOM, 2001.

[17] S. Leinen, M. Przybylski, V. Reijs, Sz. Trocha, Testing of Traffic Measurement Tools, GEANT Report GEA-01-113, October 2001.

[18] A. Markopoulou, F. Tobagi, M. Karam, Assessment of VoIP quality over Internet backbones, IEEE INFOCOM, 2002.

[19] J. Mullin, L. Smallwood, A. Watson, G. Wilson, New techniques for assessing audio and video quality in real-time interactive communications, IHM-HCI Tutorial, 2001.

[20] J. Padhye, V. Firoiu, D. Towsley, J. Kurose, Modeling TCP Throughput: A Simple Model and its Empirical Validation, 
Proc. SIGCOMM Symp. Communications Architectures and Protocols Aug. 1998, 304-314.

[21] A. Sukhov, P. Calyam, W. Daly, A. Iliin, Network requirements for high-speed real-time multimedia application, III IPv6 global Summit (Internet. New Generation -IPv6), Moscow, November 2004, p. 2833.
[22] Y. Zhu, A Survey of Network Requirements to Support Current and Future Data Streams, CS 7001 Introduction to Graduate Studies Mini-project 1, Fall 2000.

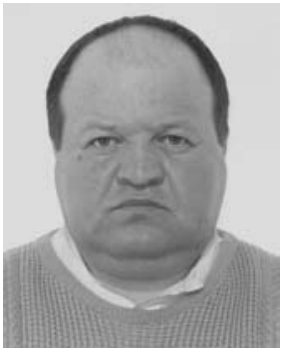

Andrei Sukhov is an Associate Professor, Head of Laboratory of Network Technologies, Samara State Academy of TransportEngineering, Russia and was awarded a PhD in Moscow, in Physics and Mathematics in 1993. Over the last 10 years he has been involved in acting as an investigator for more then 10 telecommunication projects supported by the Russian government, INTAS, NATO, ESA, US Information Agency, etc. These are the construction of Samara Regional Network for Science and Education - the first Russian provincial network providing a digital connection to Moscow, the regional transport network at level STM16, etc. He is a founder of the biggest Regional ISP and an expert in the area of telecommunication investments in Russia.

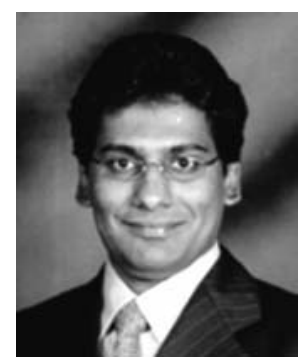

Prasad Calyam is a Systems Developer/Engineer at OARnet, A Division of The Ohio State University. He obtained his M.S. in Electrical and Computer Engineering from The Ohio State University in 2002 and is currently enrolled in the Ph.D. program in the same department. His expertise is in the design and development of software systems comprising of advanced architectures and network-based performance analysis techniques and tools. He has previously worked on projects for companies such as Mphasis, Samsung Electronics Corporation, Korea and Eaton Corporation, USA in the commercial sector and for research groups at the Indian Institute of Science, The Ohio State University, OARnet and Internet2 in the academic sector.

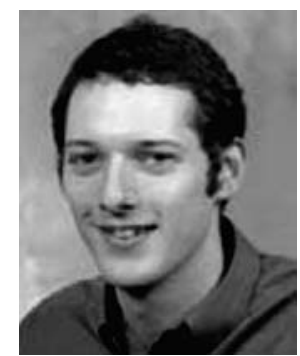

Warren Daly works as a Network Security Expert for HEAnet Ltd. He has been an IT professional for 8 years and holds a BSc in Business and Information Technology from Trinity College Dublin and a Diploma in Electronic Systems from Dublin Institute of Technology. He has successfully deployed a PKI infrastructure for the Irish Academicl\&Research community and has extensive knowledge in Information Security Management Systems (ISO17799). He is also a member of the EU National Research Networks Policy Committee Review panel.

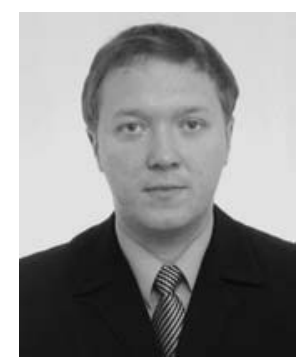

Alexander Iliin works as Network Engineer at Russian Institute for Public Networks. He graduated Moscow Institute of Physics and Technology at 1998 as engineer-physicist with specialization Telecommunication Network and Systems. He is primary working under building internetworks and monitoring systems. He is participating in different network projects for educational and commercial needs in Russia. Some of them: RBNET (Russian Backbone Network), that has been established to provide Internet service for science and high school community at main regions of Russia, RELARN (association of scientific and educational organizations) and others. He is working at IT business over 10 years and have a great experience of building and supporting global networks. He is also certified specialist Juniper, Cisco and Unix administration. 